\title{
On a Hsu-unified Structure Manifold with a Quarter- symmetric Non-metric Connection
}

\author{
Ram Nivas ${ }^{1}$ and Anurag Agnihotri ${ }^{2}$
}

Department of Mathematics and Astronomy, University of Lucknow, Lucknow-226007, India

Keywords: Quarter-symmetric non-metric connection, Hsu-unified structure manifold, Hsu- Kahler manifold, Nijenhuis tensor, Contravariant almost analytic vector field.

\begin{abstract}
In the present paper, we have defined a Hsu-unified structure manifold and a Hsu-Kahler manifold and studied some properties of the quarter-symmetric non-metric connection. Certain interesting results on such manifolds have been obtained. We have also studied the properties of the contravariant almost analytic vector field on these manifolds equipped with the quarter-symmetric non-metric connection.
\end{abstract}

\section{Introduction}

Quarter-symmetric linear connection was introduced and studied by S. Golab[1] in 1975.Several properties of quarter-symmetric metric and non-metric connections on a differentiable manifold have been studied by Yano and Imai[10], Sular etal. [8], Sengupta and Biswas[7] and many other geometors. In the present paper, we have studied some properties of the quarter-symmetric nonmetric connection on a manifold called Hsu-unified structure manifold and a Hsu- Kahler manifold which is a particular case of Hsu-unified structure manifold satisfying a certain condition. It has been shown that the Nijenhuis tensor with respect to quarter-symmetric non-metric connection $\nabla$ and with respect to Riemannian connectionD coincide in the Hsu-unified structure manifold but in the Hsu- Kahler manifold Nijenhuis tensor with respect to $\nabla$ vanishes identically i.e. a Hsu- Kahler manifold is integrable. It has also been proved that a contravariant almost analytic vector fieldV with respect to Riemannian connection D is also contravariant almost analytic with respect to quarter-symmetric non-metric connection $\nabla$ in the Hsu- Kahler manifold but in the Hsu-unified structure manifold, it is possible with a specific condition

\section{Preliminaries}

If on an even dimensional differentiable manifold $M_{n}, n=2 m$ of differentiability class $C^{\infty}$, there exists a vector valued real linear function $F$ of differentiability class $C^{\infty}, \quad$ satisfying

$$
F^{2} X=a^{r} X
$$

for arbitrary vector field $X$. Also there exists a Riemannian metric $g$, such that

$$
g(\bar{X}, \bar{Y})=a^{r} g(X, Y)
$$

where $\bar{X}=F X, 0 \leq r \leq n$ and $a$ is a real or complex number.

Then in view of the equations (2.1) and (2.2), $M_{n}$ is said to be a Hsu-unified structure manifold.

Let us define a 2 -form ' $F$ in $M_{n}$, given as

$$
{ }^{\prime} F(X, Y) g(\bar{X}, Y)=g(X, \bar{Y})
$$

Then it is clear that the 2-form ' $F$ satisfies

$$
{ }^{\prime} F(\bar{X}, \bar{Y})=a^{r \prime} F(X, Y)
$$


From (2.3), we can also conclude the following properties

$$
\begin{aligned}
& { }^{\prime} F(\bar{X}, Y)=a^{r} g(X, Y) \\
& { }^{\prime} F(X, Y)={ }^{\prime} F(Y, X)
\end{aligned}
$$

The equation (2.6) shows that the 2-form ' $F$ is symmetric in $M_{n}$. If the Hsu-unified structure manifold $M_{n}$ satisfies a condition

$$
\left(D_{X} F\right) Y=0
$$

Then $M_{n}$ will be said to be a Hsu-K $\ddot{a}$ hler manifold.

From the equation (2.7), it can be seen that

$$
D_{X} \bar{Y}-\overline{D_{X} Y} \Leftrightarrow \overline{D_{X} \bar{Y}}=a^{r}\left(D_{X} Y\right)
$$

\section{Quarter-symmetric Non-metric Connection}

A linear connection $\nabla$ defined as [9]

$$
\nabla_{X} Y=D_{X} Y+u(Y) \bar{X}
$$

or arbitrary vector fields $\mathrm{X}$ and $\mathrm{Y}$, is said to be a quarter-symmetric non-metric connection if the torsion tensorS of the connection $\nabla$ and the metric tensorg are given by

and

$$
S(X, Y)=u(Y) \bar{X}-u(X) \bar{Y}
$$

$$
\left(\nabla_{X} g\right)(\mathrm{Y}, \mathrm{Z})=-u(Y) g(\bar{X}, Z)-u(Z) g(\bar{X}, Y)
$$

where $u$ is 1 -form associated with the vector field $U$ such that

and $D$ is the Riemannian connection.

$$
u(X)=g(X, U)
$$

If we put (3.1) as

$$
\nabla_{X} Y=D_{X} Y+H(X, Y)
$$

Then

$$
H(X, Y)=u(Y) \bar{X}
$$

Let us define

$$
' H(X, Y, Z)=g(H(X, Y), Z)
$$

By virtue of the equation (3.6), (3.7) becomes

$$
{ }^{\prime} H(X, Y, Z)=u(Y) g(\bar{X}, Z)
$$

\section{Hsu-unified structure manifold equipped with the Quarter-symmetric non-metric connection}

In this section, we have the following theorems

Theorem 4.1: For a Hsu-unified structure manifold n $\mathrm{M}$ equipped with a quarter-symmetric nonmetric connection $\nabla$, the following results hold good

$$
\begin{array}{ll}
\text { (i) } H(\bar{X}, Y)=a^{r} H(X, \bar{Y}) & \text { iff } u(Y) X=u(\bar{Y}) \bar{X} \\
\text { (ii) } H(\bar{X}, Y)=H(\bar{Y}, X) \quad \text { iff } u(Y) X=u(X) Y \\
\text { (iii) } H(\bar{X}, \bar{Y})=a^{r} H(X, Y) \text { iff } u(\bar{Y}) X=u(Y) \bar{X}
\end{array}
$$


Proof: From the equations (3.6) and (2.1), we have

$$
\begin{aligned}
& \text { (i) } H(\bar{X}, Y)=a^{r} u(Y) X \\
& \text { (ii) } H(X, \bar{Y})=u(\bar{Y}) \bar{X}
\end{aligned}
$$

Clearly (4.1) ) (i) follows from (4.2)) (i and (4.2)) (ii .

Interchanging $X$ and $Y$ in (3.6), we get

$$
H(Y, X)=u(X) \bar{Y}
$$

Barring $Y$ in (4.3) and using (2.1), we have

$$
H(\bar{Y}, X)=a^{r} u(X) Y
$$

Now the result of (4.1) ) (ii) follows from (4.2) ) (i) and (4.4).

Again barring $\mathrm{X}$ and $\mathrm{Y}$ in (3.6) and using (2.1), we obtain

$$
H(\bar{X}, \bar{Y})=a^{r} u(\bar{Y}) X
$$

By virtue of the equations (3.6) and (4.5), the result of (4.1) ) (iii) follows.

Theorem 4.2: In a Hsu-unified structure manifold $\mathrm{M}_{\mathrm{n}}$ with a quarter-symmetric non-metric connection $\nabla$, we have

$$
\begin{aligned}
& \text { (i) ' } H(\bar{X}, \bar{Y}, Z)=' F(\bar{X}, Z) u(\bar{Y})=' H(X, \bar{Y}, \bar{Z}) \\
& (i i)^{\prime} H(\bar{X}, \bar{Y}, \bar{Z})=' F(\bar{X}, \bar{Z}) u(\bar{Y})=' H(\bar{Z}, \bar{Y}, \bar{X})
\end{aligned}
$$

Proof: BarringX andY in (3.8) and using the equations (2.3) and (2.5), we get

$$
\text { ' } H(\bar{X}, \bar{Y}, Z)=' F(\bar{X}, Z) u(\bar{Y})
$$

Again barring $Y$ and $Z \quad$ in (3.8) and using the equations (2.3) and (2.5), we obtain

$$
\text { ' } H(X, \bar{Y}, \bar{Z})=' F(\bar{X}, Z) u(\bar{Y})
$$

Hence the equations (4.7) and (4.8) prove the result of (4.6) ) (i) .

BarringZ in (4.7), we have

$$
\text { ' } H(\bar{X}, \bar{Y}, \bar{Z})=' F(\bar{X}, \bar{Z}) u(\bar{Y})
$$

Interchanging $\bar{X}$ and $\bar{Z}$ in (4.8), we get

$$
\text { ' } H(\bar{Z}, \bar{Y}, \bar{X})=' F(\bar{Z}, \bar{X}) u(\bar{Y})
$$

In view of the equations (4.8), (4.9) and (2.6), the result of (4.6) ) (ii) follows easily

Theorem 4.3: A Hsu-unified structure manifold $M_{n}$ with a quarter-symmetric non-metric Connection $\nabla$ satisfies the following relation 


$$
\left(\nabla_{\bar{X}} F\right) \bar{Y}=-a^{r}\left(\nabla_{X} F\right) Y \quad \text { iff }\left(D_{\bar{X}} F\right) \bar{Y}=-a^{r}\left(D_{X} F\right) Y
$$

Proof: Replacing $Y$ by $\bar{Y}$ in (3.1), we have

$$
\nabla_{X} \bar{Y}=D_{X} \bar{Y}+u(\bar{Y}) \bar{X}
$$

The above equation can also be modified as

$$
\left(\nabla_{X} F\right) Y=D_{X} \bar{Y}+u(\bar{Y}) \bar{X}-\overline{\nabla_{X} Y}
$$

Operation of $F$ on both sides of the equation (3.1) yields

$$
\overline{\nabla_{X} Y}=\overline{D_{X} Y}+a^{r} u(Y) X
$$

From the equations (4.12) and (4.13), we get

$$
\left(\nabla_{X} F\right) Y=\left(D_{X} F\right) Y+u(\bar{Y}) \bar{X}-a^{r} u(Y) X
$$

Barring $X$ and $Y$ in (4.14), we have

$$
\left(\nabla_{\bar{X}} F\right) \bar{Y}=\left(D_{\bar{X}} F\right) \bar{Y}+a^{2 r} u(Y) X-a^{r} u(\bar{Y}) \bar{X}
$$

Subtraction of the equations (4.14) and (4.15) gives

$$
\left(\nabla_{\bar{X}} F\right) \bar{Y}-\left(\nabla_{X} F\right) Y=\left(D_{\bar{X}} F\right) \bar{Y}-\left(D_{X} F\right) Y+\left(a^{r}+1\right)\left[a^{r} u(Y) X-u(\bar{Y}) \bar{X}\right]
$$

Now in view of the equations (4.14) and (4.16), we obtain

$$
\left(\nabla_{\bar{X}} F\right) \bar{Y}+a^{r}\left(\nabla_{X} F\right) Y=\left(D_{\bar{X}} F\right) \bar{Y}+a^{r}\left(D_{X} F\right) Y
$$

Clearly, the equation (4.17) proves the statement of the theorem.

Theorem 4.4: If a Hsu-unified structure manifold $\mathrm{M}_{\mathrm{n}}$ admits a quarter-symmetric non-metric Connection $\nabla$, then the Nijenhuis tensors of the Riemannian connection $\mathrm{D}$ and $\nabla$ coincide.

Proof: From the equation (4.14), we have

$$
\left(D_{X} F\right) Y=\left(\nabla_{X} F\right) Y-u(\bar{Y}) \bar{X}+a^{r} u(Y) X
$$

Replacing $X$ by $\bar{X}$ in (4.18), we get

$$
\left(D_{\bar{X}} F\right) Y=\left(\nabla_{\bar{X}} F\right) Y-a^{r} u(\bar{Y}) X+a^{r} u(Y) \bar{X}
$$

Interchanging $X$ and $Y$ in (4.19), we find

$$
\left(D_{\bar{Y}} F\right) X=\left(\nabla_{\bar{Y}} F\right) X-a^{r} u(\bar{X}) Y+a^{r} u(X) \bar{Y}
$$

Operating both sides of the equation (4.18) by $F$, we obtain

$$
\overline{\left(D_{X} F\right) Y}=\overline{\left(\nabla_{X} F\right) Y}-a^{r} u(\bar{Y}) X+a^{r} u(Y) \bar{X}
$$

Again interchanging $X$ and $Y$ in the above equation, we have

$$
\overline{\left(D_{Y} F\right) X}=\overline{\left(\nabla_{Y} F\right) X}-a^{r} u(\bar{X}) Y+a^{r} u(X) \bar{Y}
$$

The Nijenhuis tensor with respect to $F$ is a vector valued bilinear function, defined as [2], [3]

$$
N(X, Y)=[\bar{X}, \bar{Y}]-[\overline{\bar{X}, Y]}-\overline{[X, \bar{Y}]}+\overline{\overline{[X, Y]}}
$$

In view of the equation (2.1), the above expression can be written in the form 


$$
N(X, Y)=[\bar{X}, \bar{Y}]-\overline{[\bar{X}, Y]}-\overline{[X, \bar{Y}]}+a^{r}[X, Y]
$$

The Nijenhuis tensor with respect to the Riemannian connection $\mathrm{D}$ is given as

$$
N(X, Y)=\left(D_{\bar{X}} F\right) Y-\left(D_{\bar{Y}} F\right) X-\overline{\left(D_{X} F\right) Y}+\overline{\left(D_{Y} F\right) X}
$$

By virtue of the equations (4.19), (4.20), (4.21) and (4.22), (4.23) becomes

$$
\begin{aligned}
N(X, Y) & =\left(\nabla_{\bar{X}} F\right) Y-\left(\nabla_{\bar{Y}} F\right) X-\overline{\left(\nabla_{X} F\right) Y}+\overline{\left(\nabla_{Y} F\right) X} \\
& =N^{*}(X, Y)
\end{aligned}
$$

Where $N^{*}(X, Y)$ denotes the Nijenhuis tensor with respect to the quarter-symmetric non-metric connection $\nabla$. Hence we have the statement of the theorem.

\section{Hsu-Kahler manifold with the Quarter-symmetric non-metric connection}

As discussed earlier that if the Hsu-unified structure manifold $M_{n}$ satisfies the condition (2.7), then $\mathrm{M}_{\mathrm{n}}$ is called a Hsu-Kahler manifold. In this section, we have some following theorems

Theorem 5.1: If $M_{n}$ be a Hsu-Kahler manifold admitting a quarter-symmetric non-metric connection $\nabla$, then in $\mathrm{M}_{\mathrm{n}}$

$$
\begin{aligned}
& \text { (i) }\left(\nabla_{\bar{X}} F\right) \bar{Y}=-a^{r}\left(\nabla_{X} F\right) Y \\
& (\text { ii })\left(\nabla_{X} F\right) Y=0 \quad \text { iff } u(\bar{Y}) \bar{X}=a^{r} u(Y) X
\end{aligned}
$$

Proof: In a Hsu-Kahler manifold $\mathrm{n} \mathrm{M}$, the equation (4.11) can be written as

$$
\left(\nabla_{X} F\right) Y=\overline{D_{X} Y}+u(\bar{Y}) \bar{X}-\overline{\nabla_{X} Y}
$$

From the equations (5.2) and (4.13), it is clear that

$$
\left(\nabla_{X} F\right) Y=u(\bar{Y}) \bar{X}-a^{r} u(Y) X
$$

Barring $\mathrm{X}$ and $\mathrm{Y}$ in (5.3) and using (2.1), we get

$$
\left(\nabla_{\bar{X}} F\right) \bar{Y}=-a^{r}\left[u(\bar{Y}) \bar{X}-a^{r} u(Y) X\right]
$$

By virtue of the equations (5.3) and (5.4), the required result of (5.1) ) (i) follows.

Also in view of the equation (5.3), (5.1) ) (ii) is obvious.

Theorem 5.2: A Hsu-Kahler manifold $M_{n}$ with a quarter-symmetric non-metric connection $\nabla$ satisfies the following relation

$$
d^{\prime} F(X, Y, Z)=-2[u(X) g(\bar{Y}, \bar{Z})+u(Y) g(\bar{Z}, \bar{X})+u(Z) g(\bar{X}, \bar{Y})]
$$

Proof: By definition, we know that

$$
d^{\prime} F(X, Y, Z)=\left(\nabla_{X}^{\prime} F\right)(Y, Z)+\left(\nabla_{Y}^{\prime} F\right)(Z, X)+\left(\nabla_{Z}^{\prime} F\right)(X, Y)
$$

From the equation (2.3), we have

$$
\text { ' } F(Y, Z)=g(\bar{Y}, Z)
$$

Differentiating the equation (5.7) covariantly with respect to $\mathrm{X}$ and using the expressions of the equations (2.2), (2.3), (3.1) and (3.3), we obtain

$$
\left(\nabla_{X}^{\prime} F\right)(Y, Z)=-[u(Z) g(\bar{X}, \bar{Y})+u(Y) g(\bar{Z}, \bar{X})]
$$

Proceeding in the same way, we can also get

$$
\begin{aligned}
& \left(\nabla_{Y}{ }^{\prime} F\right)(Z, X)=-[u(X) g(\bar{Y}, \bar{Z})+u(Z) g(\bar{X}, \bar{Y})] \\
& \left(\nabla_{Z}{ }^{\prime} F\right)(X, Y)=-[u(Y) g(\bar{Z}, \bar{X})+u(X) g(\bar{Y}, \bar{Z})]
\end{aligned}
$$

In consequence with the equations (5.6), (5.8), (5.9) and (5.10), the required result follows. 
Theorem 5.3: The Nijenhuis tensor with respect to the quarter-symmetric non-metric connection $\nabla$ in a Hsu-Kahler manifold $M_{n}$ vanishes i.e. the manifold $M_{n}$ is integrable.

Proof: The Nijenhuis tensor with respect to the quarter-symmetric non-metric connection $\nabla$ is defined as

$$
N^{*}(X, Y)=\left(\nabla_{\bar{X}} F\right) Y-\left(\nabla_{\bar{Y}} F\right) X-\overline{\left(\nabla_{X} F\right) Y}+\overline{\left(\nabla_{Y} F\right) X}
$$

Replacing $X$ by $\bar{X}$ in (5.3), we get

$$
\left(\nabla_{\bar{X}} F\right) Y=a^{r}[u(\bar{Y}) X-u(Y) \bar{X}]
$$

Interchanging $X$ and $Y$ in (5.12), we obtain

$$
\left(\nabla_{\bar{Y}} F\right) X=a^{r}[u(\bar{X}) Y-u(X) \bar{Y}]
$$

Operation of $\mathrm{F}$ on both sides of the equation (5.3) yields

$$
\left.\overline{(\nabla}{ }_{X} F\right) Y=a^{r}[u(\bar{Y}) X-u(Y) \bar{X}]
$$

Interchanging $\mathrm{X}$ and $\mathrm{Y}$ in (5.14), we have

$$
\overline{\left(\nabla_{Y} F\right) X}=a^{r}[u(\bar{X}) Y-u(X) \bar{Y}]
$$

In view of the equations $(5.12),(5.13),(5.14)$ and (5.15), the equation (5.11) takes the form

which proves the theorem.

$$
N^{*}(X, Y)=0
$$

\section{Contravariant almost analytic vector field}

A vector field $\mathrm{V}$ is said to be contravariant almost analytic if the Lie-derivative of the tensor field $\mathrm{F}$ with respect to $\mathrm{V}$ vanishes identically i.e. [2], [3]

$$
\left(L_{V} F\right) X=0 \text { for all } X
$$

The equation (6.1) can also be written as

$$
[V, \bar{X}]=\overline{[V, X]}
$$

In a Hsu-unified structure manifold, the equation (6.2) takes the form

$$
\left(D_{V} F\right) X-D_{\bar{X}} V+\overline{D_{X} V}=0
$$

In a Hsu-K $\ddot{a}$ hler manifold, the equation (6.2) becomes

$$
D_{\bar{X}} V-\overline{D_{X} V}=0 \Leftrightarrow \overline{D_{\bar{X}} V}=a^{r}\left(D_{X} V\right)
$$

Now we have the following theorems

Theorem 6.1: On a Hsu-unified structure manifold, a contravariant almost analytic vector field V with respect to the Riemannian connection D is also contravariant almost analytic with respect to the quarter-symmetric non-metric connection $\nabla$ if

$$
u(\bar{X}) \bar{V}=a^{r} u(X) V
$$

Proof: For any vector field V, the equation (3.1) takes the form

$$
\nabla_{X} V=D_{X} V+u(V) \bar{X}
$$

Replacing $X$ by $\bar{X}$ in (6.5), we have

$$
\nabla_{\bar{X}} V=D_{\bar{X}} V+a^{r} u(V) X
$$


Operating both sides of the equation (6.5) by $\mathrm{F}$, we get

$$
\overline{\nabla_{X} V}=\overline{D_{X} V}+a^{r} u(V) X
$$

Interchanging $X$ and $V$ in (6.5), we obtain

$$
\nabla_{V} X=D_{V} X+u(X) \bar{V}
$$

From (6.8), it is clear that

$$
\begin{aligned}
& \text { (i) } \nabla_{V} \bar{X}=D_{V} \bar{X}+u(\bar{X}) \bar{V} \\
& \text { (ii) } \overline{\nabla_{V} X}=\overline{D_{V} X}+a^{r} u(X) V
\end{aligned}
$$

As we know that

$$
\left(\nabla_{V} F\right) X=\nabla_{V} \bar{X}-\overline{\nabla_{V} X}
$$

In consequence with the equation (6.9), the last expression becomes

$$
\left(\nabla_{V} F\right) X=D_{V} \bar{X}-\overline{D_{V} X}+u(\bar{X}) \bar{V}-a^{r} u(X) V
$$

Now by virtue of the equations (6.6), (6.7) and (6.10), we find that

$$
\left(\nabla_{V} F\right) X-\nabla_{\bar{X}} V+\overline{\nabla_{X} V}=\left(D_{V} F\right) X-D_{\bar{X}} V+\overline{D_{X} V}+u(\bar{X}) \bar{V}-a^{r} u(X) V
$$

Since $\mathrm{V}$ is contravariant almost analytic vector field with respect to the Riemannian connection $\mathrm{D}$, so using (6.3), the equation (6.11) is transformed into

$$
\left(\nabla_{V} F\right) X-\nabla_{\bar{X}} V+\overline{\nabla_{X} V}=u(\bar{X}) \bar{V}-a^{r} u(X) V
$$

If the vector field $\mathrm{V}$ is also contravariant almost analytic with respect to the quarter-symmetric nonmetric connection $\nabla$, then it will satisfy

$$
\left(\nabla_{V} F\right) X-\nabla_{\bar{X}} V+\overline{\nabla_{X} V}=0
$$

Hence the equation (6.12) proves the statement of the theorem.

Theorem 6.2: On a Hsu-Kahler manifold, a contravariant almost analytic vector field V with respect to the Riemannian connectionD is also contravariant almost analytic with respect to the quarter-symmetric non-metric connection $\nabla$.

Proof: Subtraction of the equation (6.7) from (6.6) gives

$$
\nabla-\bar{X}-\overline{\nabla_{X} V}=D_{\bar{X}} V-\overline{D_{X} V}
$$

Since $\mathrm{V}$ is contravariant almost analytic vector field with respect to the Riemannian connection D in the Hsu-Kahler manifold, so with the help of the equation (6.4), (6.13) yields

$$
\nabla_{\bar{X}} V-\overline{\nabla_{X} V}=0
$$

which implies that $\mathrm{V}$ is also contravariant almost analytic vector field with respect to the quartersymmetric non-metric connection $\nabla$.

\section{References}

[1] Golab, S. (1975): On semi-symmetric and quarter-symmetric linear connections, Tensor N. S., Vol. 29, pp. 249-254.

[2] Kobayashi, S. and Nomizu, K. (1969): Foundation of differential geometry, Vol. I and II, Interscience Publisher, London.

[3] Mishra, R. S. (1984): Structures on a differentiable manifold and their applications, Chandrama Prakashan, 50-A Balrampur House, Allahabad, India. 
[4] Mondal, A. K. and De, U. C. (2009): Some properties of a Quarter-symmetric metric connection on a Sasakian manifold, Bull. Math. Anal. Appl., Vol. 1, Issue 3, pp. 99-108.

[5] Mukhopadhyay, S., Roy, A. K. and Barua, B. (1991): Some properties of a quarter- symmetric metric connection on a Riemannian manifold, Soochow J. of Math., Vol. 17 (2),pp. 205-211.

[6] Singh, B. P. (2005): Hypersurfaces of a unified structure manifold, Acta Ciencia Indica, Vol. XXXI (2), pp. 487-490.

[7] Sengupta, J. and Biswas, B. (2003): Quarter-symmetric non-metric connection on a Sasakian manifold, Bull. Cal. Math. Soc., Vol. 95(2), pp. 169-176.

[8] Sular, S., Ozgur, C. and De, U. C. (2008): Quarter-symmetric metric connection in a Kenmotsu manifold, SUT. J. Math., Vol. 44 (2), pp. 297-306.

[9] Singh, R. N. and Pandey, M. K. (2007): On a type of quarter-symmetric non-metric connection in a Kenmotsu manifold, Bull. Cal. Math. Soc., vol. 99 (4), pp. 433-444.

[10] Yano K. and Imai, T. (1982): Quarter-symmetric metric connections and their curvature tensors, Tensor N. S., Vol. 38, pp. 13-18. 